\title{
Multiparametric Porcine Oocyte Deformation Characterization by Novel MEMS-Type Cytometer ${ }^{\dagger}$
}

\author{
Aleksandra Pokrzywnicka 1,*, Danylo Lizanets ${ }^{1}$, Patrycja Śniadek ${ }^{2}$, Natalia Małyszka ${ }^{2}$ and \\ Rafał Walczak ${ }^{1}$ \\ 1 Department of Microsystem Electronics and Photonics, Wroclaw University of Science and Technology, \\ 50-370 Wroclaw, Poland; danylo.lizanets@pwr.edu.pl (D.L.); rafal.walczak@pwr.edu.pl (R.W.) \\ 2 Department of Veterinary Medicine and Animal Sciences, Poznan University of Life Sciences, 60-637 Poznan, \\ Poland; patrycja.sniadek@pwr.edu.pl (P.Ś.); malyszka@up.poznan.pl (N.M.) \\ * Correspondence: aleksandra.pokrzywnicka@pwr.edu.pl; Tel.: +48-320-4994 \\ † Presented at the Eurosensors 2018 Conference, Graz, Austria, 9-12 September 2018.
}

Published: 29 December 2018

\begin{abstract}
In this work for the first time MEMS-type deformation microcytometer and a new methodology for the determination of cell deformability is proposed. Novel chip design and methodology allow real-time observation of the cell deformation occurring during compression of oocytes. In addition, software-based analysis of microscopic images allows multiparametric characterization of the investigated cell toward quality assessment of the cell.
\end{abstract}

Keywords: mechanical properties of cells; deformability of oocytes; microcytometry; microengineering; MEMS

\section{Introduction}

Cells change their mechanical properties due to interaction with the elements of the environment, or reaction to stimuli located in it. Mechanical properties of cells may be used as labelfree determinants of the state and biological quality of cells.

Only a few literature reports concern mechanical properties of the oocytes determined by use of MEMS devices [1,2]. Among the existing MEMS solutions, especially microfluidic structures, there is a lack of standarization due to the variety of measurement methods used and the multiplicity of determined parameters that cannot be compared with each other [3-5]. Usually only single parameter is given as result, e.g., deformation amount or deformation ratio. Oocyte has complex structure and cannot be considered as homogenous object, therefore it should be described more precisely by multiparametric characterization. There is a need for new technical and methodological solutions for the study of reproductive cells mechanobiology, including mainly oocytes, due to the relatively few tests carried out using miniaturized microfluidic systems. Therefore, an attempt was made to find the correlation between the mechanical properties of porcine oocytes with their quality of reproductive functions.

\section{Materials and Methods}

The MEMS-type deformation microcytometer consists of a microfluidic circuit fabricated in a glass substrate and a micromechanical system made in a single crystal silicon substrate anodically bonded each to other. The examined cell is introduced through a hole in the upper glass substrate into fluidic microchannel, and then it is directed to the measurement chamber and positioned in its central part directly above bossed area of the silicon membrane (Figures 1 and 2). 


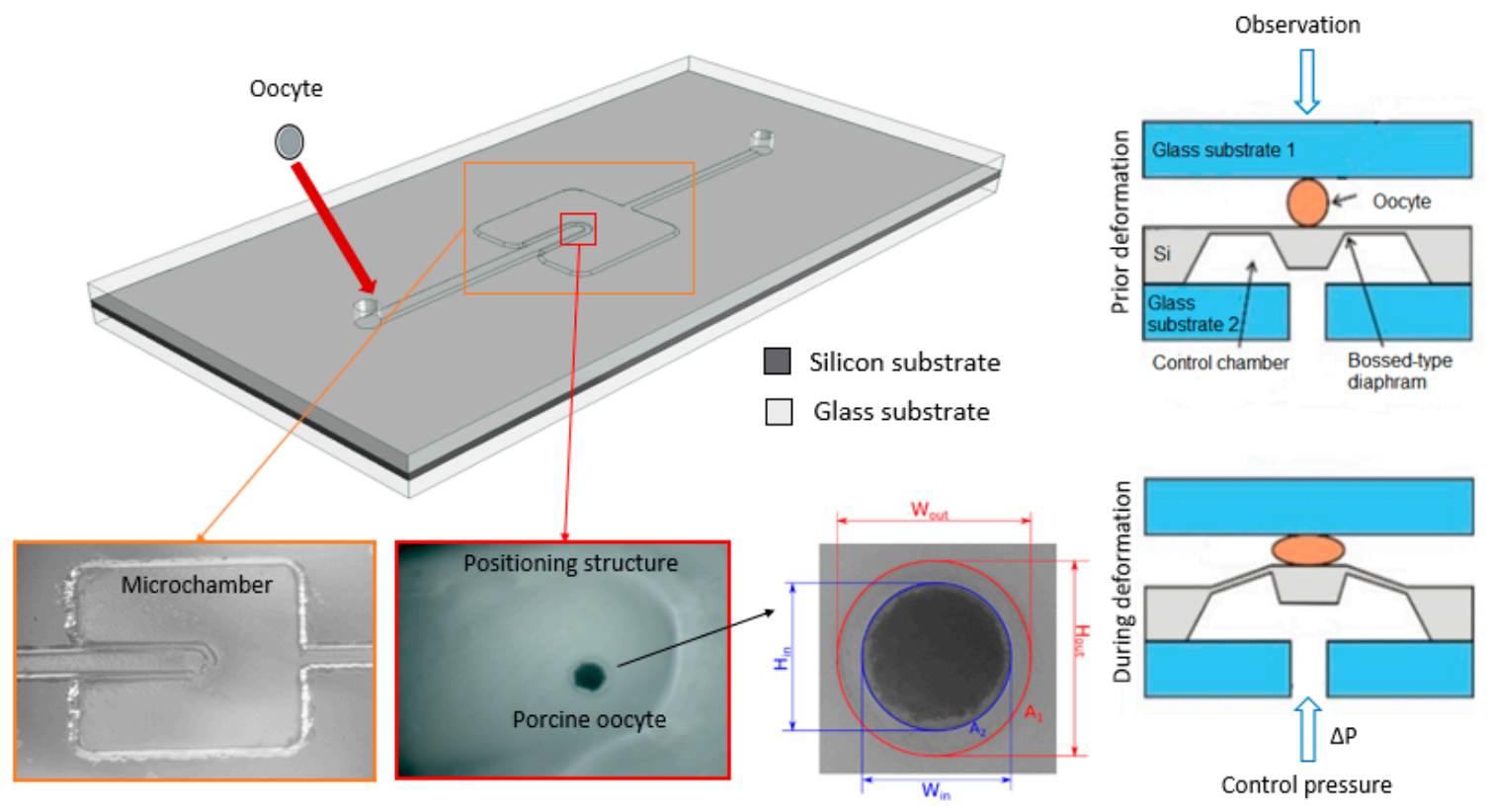

Figure 1. Visualization of the deformation micrometer (view from above) along with microscopic photo of the micromachined trapping structure in the glass, next view of the porcine oocyte in the measurement chamber and cell with the parameters determined by the software analysis ( $\mathrm{W}_{\text {out, }} \mathrm{H}_{\text {out, }}$ $\left.\mathrm{W}_{\text {in, }} \mathrm{H}_{\text {in, }} \mathrm{A}_{1}, \mathrm{~A}_{2}\right)$.

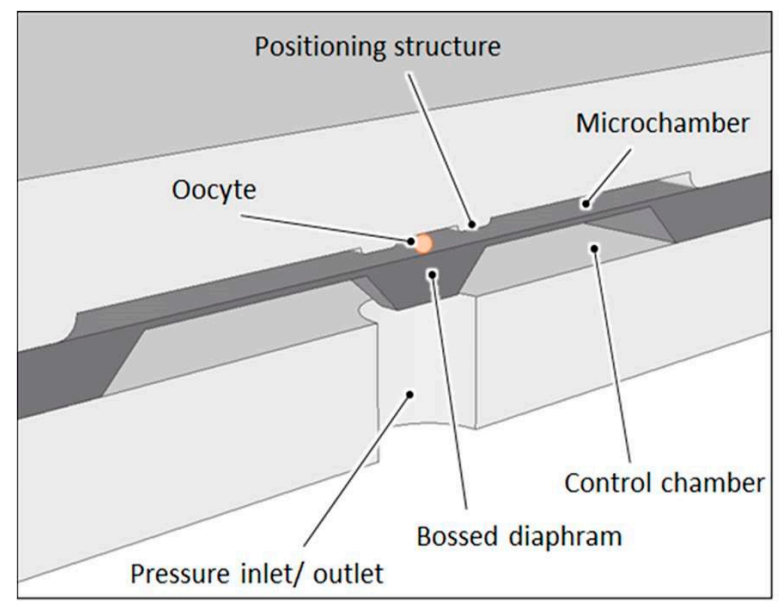

Figure 2. Schematic cross section through the microcytometer structure with trapped oocyte.

The micromechanical structure that squeezes the cell is a pneumatically actuated silicon bossed diaphragm (50 $\mu \mathrm{m}$ thick, $5 \mathrm{~mm} \times 5 \mathrm{~mm}$ large). Elveflow pressure controller with an accuracy of $0.1 \mathrm{kPa}$ is used as precise pressure regulator. A microscope with camera and dedicated software is used to record images of the cell during deformation. Observation of the cell is carried out "from above" to identify detailed microstructure of the oocyte. The analysis software enables identification of the cell and its individual elements, determination of various geometrical parameters (inside and outside diameters of the cell components and surface areas of the inside structures) (see Figure 1).

\section{Results}

In the experiments 10 porcine oocytes were deformed with membrane actuated up to $100 \mathrm{kPa}$. Although all examined cells were classified as 1st class some differences and tendencies in the deformation of cells were noticed. The first measurements of cells suggest approximately linear dependence of cell diameter changes due to the membrane actuation pressure (for example cells no 1, 2 or 8) (Figure 3). 


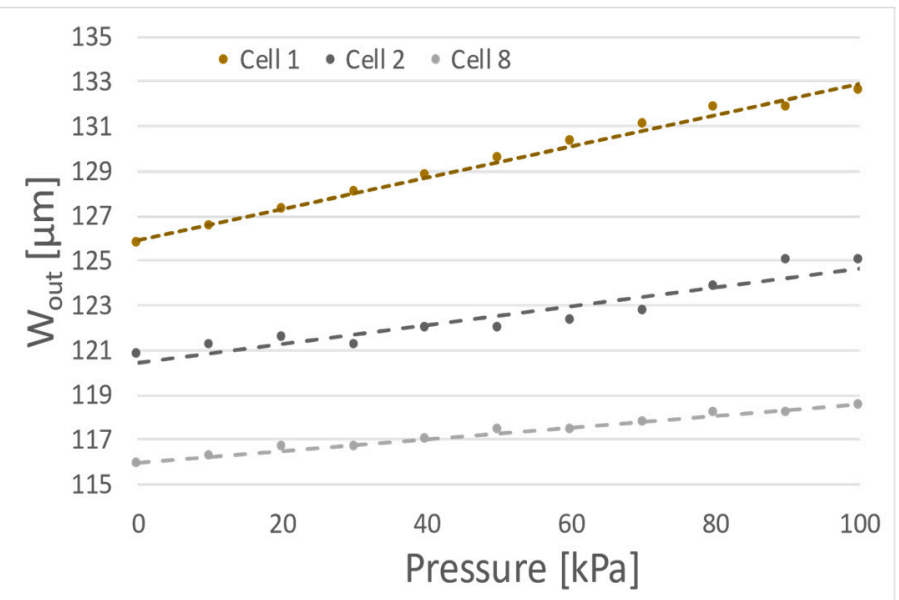

Figure 3. Exemplary dependences of the outside diameter ( $W_{\text {out }}$ ) of the oocytes as function of the membrane actuation pressure (three specific results).

We can also observe uneven cell deformation during compression. Differences in the degree of change of the diameter (external $W_{\text {out }}$ and $H_{\text {out }}$, and internal $W_{\text {in }}$ and $H_{\text {in }}$ ) show the elliptical nature of the oocytes shape (Figure 4). In addition, it seems that the that the degree of deformation of oocytoplasm' surface area $\left(\mathrm{A}_{2}\right)$ may differ from the degree of the deformation of the whole cell including the outer part of the cell-zona pellucida $\left(\mathrm{A}_{1}\right)$ (Figure 5).

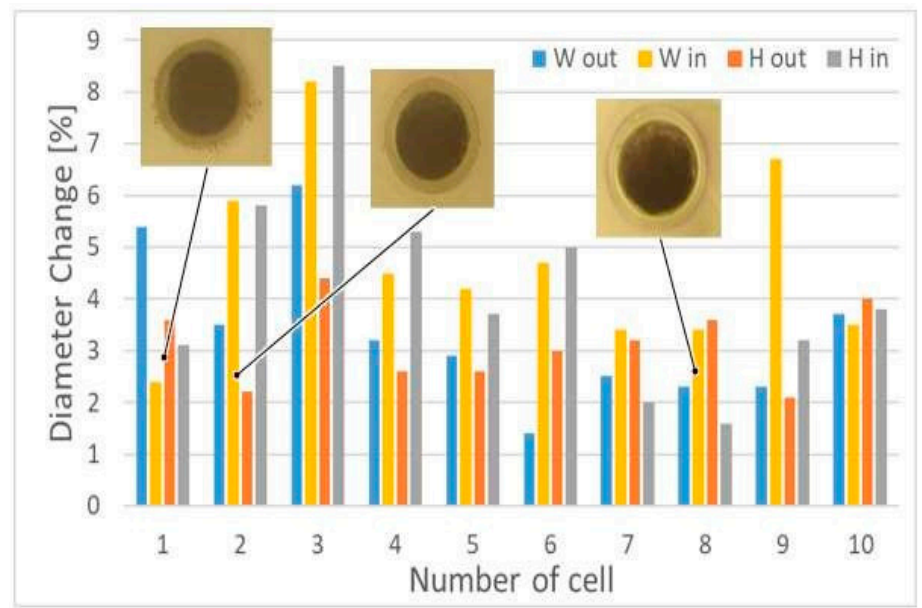

Figure 4. Dependence of the different cells' diameters change during porcine oocyte deformation; microscopic images of the selected cells, prior the deformation, are also showed.

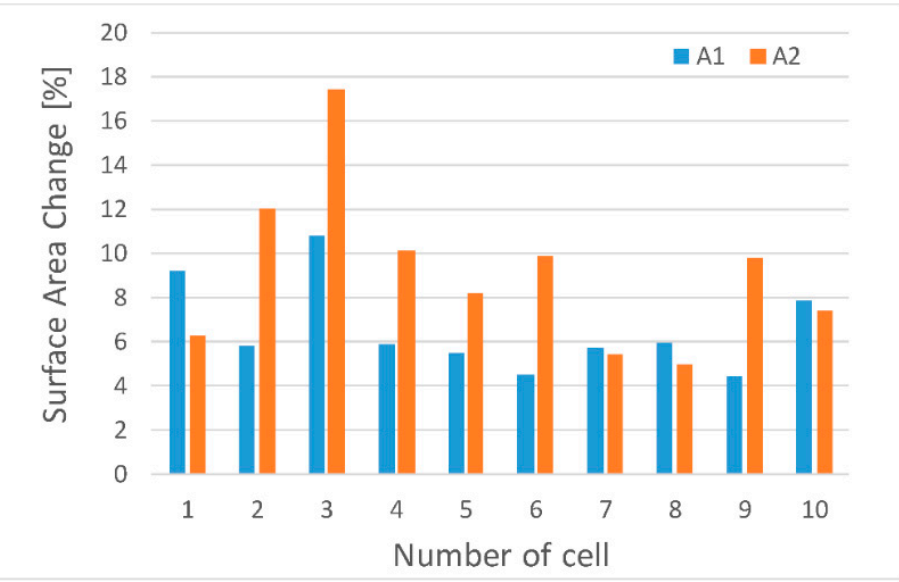

Figure 5. Dependence of the cell surface areas change during porcine oocyte deformation. 


\section{Discussion}

MEMS-type deformation microcytometer along with dedicated program allows observation of the oocyte behavior during compression and determination of cell deformability. Initial experiments show the complexity of oocytes deformation and differences between cells within the 1st class of quality. Determined parameters and related to them tendencies have been identified for the first time and may lead to definition of quality sub-groups under the same quality class in the future.

Author Contributions: R.W. and A.P. conceived and designed the experiments, A.P., P.Ś. and N.M. performed the experiments, D.L. wrote a program dedicated to the experiments carried out, A.P., P.Ś. and R.W. analyzed the data, A.P. and R.W. wrote the paper.

Acknowledgments: Work on the MEMS deformation microcytometer is carried out as part of a project funded by the National Center for Science OPUS 10 (project No. 2015/19/B/ST8/01110). The viability test of oocytes was maintained and delivery of porcine oocytes was financed from the resources of the National Science Center granted as part of financing the internship after obtaining the doctoral degree on the basis of decision number DEC-2013/08/ S/ST7/00575.

Conflicts of Interest: The authors declare no conflict of interest. The founding sponsors had no role in the design of the study; in the collection, analyses, or interpretation of data; in the writing of the manuscript, and in the decision to publish the results.

\section{References}

1. Nakahara, K.; Sakuma, S.; Hayakawa, T.; Arai, F. On-Chip Transportation and Measurement of Mechanical Characteristics of Oocytes in an Open Environment. Micromachines 2015, 6, 648-659, doi:10.3390/mi6050648.

2. Nakahara, K.; Sakuma, S.; Arai, F. On-chip Cell Separation based on mechanical characteristics. In Proceedings of the 21st MicroTAS Conference on Miniaturized Systems for Chemistry and Life Sciences, Savannah, GA, USA, 22-26 October 2017; pp. 677-678.

3. Sun, Y.; Wan, K.T.; Roberts, K.P.; Bischof, J.C., Nelson J. B. Mechanical property characterization of mouse zona pellucida. IEEE Trans. Nanobiosci. 2003, 2, 279.

4. Luo, Z.; Guven, S.; Gozen, I.; Chen, P.; Tasoglu, S.; Anchan, R.M.; Bai, B.; Demirci, U. Deformation of a single mouse oocyte in a constricted microfluidic channel. Microfluid. Nanofluid. 2015, 19, 883-890.

5. Chan, B.P.; Li, C.H.; Au-Yeung, K.L.; Sze, K.Y.; Ngan, A.H. A microplate compression method for elastic modulus measurement of soft and viscoelastic collagen microspheres. Ann. Biomed. Eng. 2008, 36, 1254-1267.

(C) 2018 by the authors. Licensee MDPI, Basel, Switzerland. This article is an open access article distributed under the terms and conditions of the Creative Commons Attribution (CC BY) license (http://creativecommons.org/licenses/by/4.0/). 